\title{
Correction to: Cytotoxic and anti-inflammatory compounds from Red Sea grass Thalassodendron ciliatum
}

\author{
Reda F. Abdelhameed ${ }^{1,2} \cdot$ Amany K. Ibrahim ${ }^{2} \cdot$ Koji Yamada $^{1} \cdot$ Safwat A. Ahmed ${ }^{2}$
}

Published online: 9 May 2018

(c) Springer Science+Business Media, LLC, part of Springer Nature 2018

\section{Correction to: Medicinal Chemistry Research}

27:1238-1244

https://doi.org/10.1007/s00044-018-2143-7.

The original version of this article unfortunately contained a mistake in the Electronic Supplementary Material.
The typesetter has incorrectly processed the ESM and the correct ESM is provided with this erratum.

The original article can be found online at https://doi.org/10.1007/ s00044-018-2143-7.

Electronic supplementary material The online version of this article (https://doi.org/10.1007/s00044-018-2181-1) contains supplementary material, which is available to authorized users.

Safwat A. Ahmed

safwat_aa@yahoo.com

1 Graduate School of Biomedical Sciences, Nagasaki University, 114 Bunkyo-machi, Nagasaki 852-8521, Japan

2 Department of Pharmacognosy, Faculty of Pharmacy, Suez Canal University, Ismailia 41522, Egypt 